
cultures

Les cahiers de l'Acedle

15-1 | 2018

La conceptualisation grammaticale

\title{
Un point de vue rétrospectif sur les « exercices de conceptualisation »
}

\section{Henri Besse}

\section{Q OpenEdition}

Journals

Édition électronique

URL : http://journals.openedition.org/rdlc/2650

DOI : $10.4000 /$ rdlc. 2650

ISSN : 1958-5772

Éditeur

ACEDLE

Référence électronique

Henri Besse, «Un point de vue rétrospectif sur les « exercices de conceptualisation » ", Recherches en didactique des langues et des cultures [En ligne], 15-1 | 2018, mis en ligne le 02 janvier 2018, consulté le 01 mai 2019. URL : http://journals.openedition.org/rdlc/2650; DOI : 10.4000/rdlc.2650

Ce document a été généré automatiquement le 1 mai 2019.

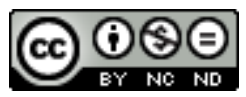

Recherches en didactique des langues et des cultures is licensed under a Creative Commons AttributionNonCommercial-NoDerivatives 4.0 International License 


\title{
Un point de vue rétrospectif sur les " exercices de conceptualisation »
}

\author{
Henri Besse
}

1 «Tout parcours scientifique comporte des moments autobiographiques ${ }^{1} »$. Proposition qui rompt avec ce qui est en général déconseillé par la doxa universitaire qui exige, à des fins justifiées de neutralité, que ces moments soient masqués ou tus. Mais une recherche intellectuelle n'échappe jamais tout à fait à la biographie de celui ou de celle qui la mène, et l'on est donc en droit de se libérer parfois de cette doxa. C'est ce que nous allons faire en revenant rétrospectivement sur les « exercices de conceptualisation » que nous avons proposés, alors dans le cadre de la méthode $\mathrm{SGAV}^{2}$, au tout début des années 1970.

2 Une proposition quelque peu innovante n'est pas dissociable des contextes (institutionnels, sociaux, idéologiques...) au sein desquels elle apparaît, contextes où elle est parfois inaperçue, parfois contestée, plus souvent mécomprise, avant qu'elle ne soit oubliée ou adoptée. Débutons par un rappel de nos activités de jeune didacticien du français «langue étrangère » (L2), alors que, recruté depuis peu au $\mathrm{CREDIF}^{3}$, notre connaissance de l'histoire de ce domaine était plus que rudimentaire.

\section{Retour, quarante ans plus tard, sur ma critique des « exercices structuraux »}

3 On distingue communément deux manières d'enseigner la "grammaire d'une langue ", entendue comme les régularités ou analogies d'ordre morpho-syntaxique qui la distinguent des autres langues. Soit l'on (le manuel ou le maître) procède déductivement, de la règle grammaticale, formulée, le plus souvent ${ }^{4}$, par le manuel ou le maitre dans la langue « maternelle » (L1) des apprenants, aux exemples qui l'illustrent et aux exceptions qui la limitent. Soit l'on procède inductivement, en partant d'exemples choisis à cet effet en fonction de la règle que l'on veut enseigner, laquelle est ensuite explicitée par le maître et/ou par ses élèves. Notons que, bien souvent, ceux-ci n'y parviennent par eux-mêmes que s'ils ont déjà appris une règle analogue à propos de leur L1 ou d'une première L2, le 
maître devant la plupart du temps les aider à la "découvrir » quand, seuls, ils n'y parviennent pas.

Il y a là une difficulté inhérente à la circularité de tout raisonnement inductif de ce type, qui a joué un rôle dans l'émergence d'une variante radicale de cette démarche inductive, connue en français sous la dénomination d'exercices structuraux. Elle consiste à faire répéter aux élèves, de manière intensive, une série d'exemples choisis par le maitre en fonction d'une règle, cette pratique étant censée permettre de l'inculquer sans que le manuel ou le maître ait à l'expliciter. C'est, lit-on parfois, faire de la grammaire implicite, un oxymoron ignoré des grammairiens et des linguistes; il serait plus exact de parler de grammaire implicitée, la règle étant alors " pliée dans ${ }^{5}$ » les exemples qui l'illustrent.

5 Les exercices structuraux, tels qu'ils se sont imposés en France dans les années 1960, relèvent typiquement de cette pratique implicitée. La dénomination qui leur fut alors donnée en français se voulait moins militaire et plus «scientifique » que l'originelle, celle des patterns drills ${ }^{6}$ nord-américains dont ils procédaient. Mais alors que ces drills étaient le plus souvent précédés d'une consigne explicitant (dans la L1 des élèves) le point grammatical traité, et suivis d'une Generalization qui en explicitait le pattern (dans cette même L1), un exercice structural se devait d'être pratiqué, tant dans les manuels qu'en classe ou au laboratoire, sans que sa structure ne soit en quelque manière ${ }^{7}$ explicitée.

Pour justifier ce psittacisme, on présentait ces exercices comme l'application de deux théories d'origine nord-américaine : celle d'un certain «structuralisme distributionnel » et celle d'un certain "béhaviorisme", lequel était fondé sur l'hypothèse que tout apprentissage, y compris celui des langues (L1 ou L2), n'était qu'une affaire d'habitudes acquises par stimuli-réponses, coupé donc de ce qui peut se passer dans la tête (la black box ou "boîte noire ») de l'apprenant. D'après ces deux théories, "appliquées » à un domaine autre que ceux pour lesquels elles avaient été élaborées, la répétition mécanique d'exemples illustrant une « structure » était ainsi censée l'inculquer, sans que l'apprenant ait à la penser. Il s'agissait, disait-on, de « l'automatiser ».

7 Parmi les premiers articles que j'ai publiés en didactique des L2, trois sont une critique (voir Besse 1970a, 1970b, 1972 ${ }^{8}$ ) de ces exercices structuraux, parce qu'ils avaient, entre autres, l'inconvénient d'inciter les élèves à surgénéraliser la structure ainsi automatisée 9 En outre, leurs références théoriques laissaient croire qu'il pouvait y avoir «structure » hors de tous sens, que ce soit ceux permettant aux natifs de la L2 de la juger admissible grammaticalement ou ceux que lui prêtaient spontanément les élèves apprenant cette L2.

\section{De la naissance des « exercices de conceptualisation »}

8 Le premier article quelque peu théorique que j'ai publié afin de proposer des exercices autres que les exercices structuraux a pour titre : «Problèmes de sens ». Sans entrer dans le détail de son argumentation, en voici la conclusion (Besse 1970a : 77) :

(C)es exercices [...] n'ont d'autres buts que de préciser et de régulariser ces compétences partielles par tout un travail pédagogique inductif, qui permet aux élèves de prendre conscience des règles sous-jacentes à leur discours, de les conceptualiser [nous soulignons] dans une formalisation faite au niveau de leur propre langage (ce qui permet de court-circuiter les terminologies grammaticales trop liées aux traditions philologiques de chaque pays), et qui leur donne ensuite la possibilité de créer de nouveaux énoncés. La grammaire se trouve ainsi réintroduite dans l'enseignement des langues [...] comme réflexion a posteriori sur des 
formes déjà acquises [...]. Mais ce travail pédagogique n'est possible que si les élèves sont sensibles au sens des énoncés transformés ou non. [...] Une théorie du sens qui permet au professeur de travailler au niveau réel de la parole, [...], dans des conditions d'énonciation restituées, semble seule répondre aux exigences d'un enseignement des langues.

Par «compétences partielles », nous entendions que certains élèves développent avant d'autres une intuition de ce qui est grammaticalement possible et de ce qui ne l'est pas en L2, non dans l'ensemble de celle-ci mais dans l'un ou l'autre de ses micro-domaines, ceux que ces élèves ont préalablement pratiqués dans des activités ayant été réellement signifiantes pour eux, activités qui partant leur ont permis de devenir « sensibles au sens » de leurs productions dans cette L2. L'idée fondatrice de ces exercices était de s'appuyer sur les « compétences partielles » de ces élèves pour aider ceux qui n'en disposaient pas encore à mieux comprendre leurs difficultés, en leur permettant « de les conceptualiser » dans « leur propre langage » et non nécessairement dans celui des grammairiens ou des linguistes.

Notons que si conceptualiser est présent dans Besse 1970a, conceptualisation n'y est pas ; il l'est entre guillemets dans Besse 1972 [écrit en 1970] avant de l'être sans guillemets dans Besse 1974. Où il est dûment précisé que si les exercices de conceptualisation ressemblent à ceux de la "grammaire inductive ", ils en diffèrent «sur plus d'un point » $(1974: 38)$. Entre autres, en ce qu'ils visent non pas à faire «apprendre une règle ou une loi de la langue ", mais à aider les élèves à formuler, " avec les moyens du bord ", l'idée qu'ils se font eux-mêmes du fonctionnement grammatical des micro-domaines qu'ils ont déjà pratiqués de manière signifiante en L2, afin qu'ils parviennent " petit à petit » à élaborer «dans la classe un code terminologique, peut-être imprécis, mais compréhensible pour l'ensemble du groupe et ayant valeur purement opératoire pour produire de nouveaux énoncés » (ibid. 43-44).

Précisons brièvement en quoi un exercice de conceptualisation diffère d'un exercice inductif traditionnel. Il ne peut être prévu à l'avance. C'est à propos d'une erreur grammaticale, répétée par certains élèves mais non par d'autres de la même classe de L2, ou d'une question d'ordre grammatical posée par l'un ou l'autre, que le maître, au lieu de répondre à cette question ou de corriger cette erreur, propose à ses élèves de rappeler ou d'inventer des phrases incluant la forme questionnée. Il se borne à leur dire celles qui sont grammaticalement possibles en L2 et celles qui ne le sont pas, ce qui permet au groupe-classe de se constituer un petit corpus d'exemples et de contre-exemples correspondant au peu qu'ils ont appris de la L2, à leur « interlangue ». Corpus qui, portant sur ce qu'ils savent déjà, stimule en général chez eux des réflexions d'ordre métalinguistique ${ }^{10}$, qui doivent beaucoup à leur "passé grammatical» (ce qu'ils ont appris de "grammaire » à propos de leur L1 ou d'une première L2), visant à expliquer la compréhension qu'ils se font de ce qui est ou non grammaticalement possible, quant au micro-domaine concerné, dans la L2 qu'ils sont en train d'apprendre. On valide ou invalide ensuite, collectivement, ces essais de "conceptualisation" sur l'ensemble des exemples et contre-exemples recensés. Si l'une d'entre elles est validée, on en teste la solidité en généralisant son application à de nouveaux exemples inventés par les élèves. Si aucune ne s'avère valide, on abandonne l'exercice, quitte à y revenir ultérieurement quand les élèves auront développé une interlangue plus avancée. Un cahier des corpus produits et des conceptualisations validées mais provisoires (elles seront amendées ou invalidées à des étapes ultérieures) peut être tenu, sorte de mémoire de la réflexion grammaticale du groupe-classe quant à son apprentissage de cette L2. 
sont, le plus souvent, les catégories et raisonnements grammaticaux qu'ils ont scolairement appris à propos de leur L1 ou d'une première L2, et que ces raisonnements et catégories ne conviennent pas toujours à la L2 qu'ils sont en train d'apprendre. Dans l'apprentissage d'une L2, les interférences ne sont pas que d'ordre linguistique, elles sont aussi d'ordre métalinguistique : les catégories grammaticales enseignées à propos de la L1 entravent parfois l'apprentissage de celles de la L2 (voir Besse, 2005). Quant aux apprenants relevant de traditions éducatives où l'on n'enseigne pas de " grammaire », ne disposant guère de "moyens du bord " à même de raisonner grammaticalement, ces exercices ne les intéressent pas. Nous reviendrons sur ce point, capital au moins pour le français L2, dans notre dernière partie (en 5.).

\section{Un point de vue « constructiviste »}

Il y avait, dans mes articles de 1970 et 1974, un non-dit qui ne transparait que dans certaines de leurs formulations. Il était lié à ma conviction d'alors que les règles ou structures des grammairiens et des linguistes ne sont pas immanentes à ce qui distingue une langue d'une autre, et que donc on ne peut les extraire par simple induction généralisante des régularités ou analogies observables dans les productions des natifs d'une langue. Ma conviction était, et est toujours, qu'elles relèvent inévitablement d'un point de vue théorique, et que, observées d'un autre point de vue théorique, ces mêmes régularités ou analogies renvoient à des règles ou des structures différentes (voir la première partie de Besse \& Porquier, 1984). En d'autres termes, on n'est à même de « découvrir » des règles que si l'on dispose au préalable d'outils conceptuels, d'ordre théorique, à même de les (re)construire. Une conviction que je m'étais faite lors de mes études de « grammaire et philologie françaises $»^{11}$, et que ma lecture de F. de Saussure et d'É. Benveniste n'avait fait que conforter. Deux remarques, incidentes à leurs propos, m'avaient particulièrement frappé.

Celle de Saussure se trouve dans le chapitre III («Objet de la linguistique ») de son Cours de linguistique générale [1915] (1973 : 23, nous soulignons) :

D'autres sciences opèrent sur des objets donnés d'avance et qu'on peut considérer ensuite à différents points de vue, dans notre domaine, rien de semblable. [...] Bien loin que l'objet précède le point de vue, on dirait que c'est le point de vue qui crée l'objet, et d'ailleurs rien ne nous dit à l'avance que l'une des manières de considérer le fait en question soit antérieure ou supérieure aux autres.

Si ce passage m'avait frappé, c'est qu'il remettait en cause une théorie de la connaissance d'ordre positiviste qui avait dominé ma formation antérieure et qui était encore dominante dans les «sciences sociales». Ne suggérait-il pas (cf. le conditionnel «on dirait ») qu'une forme de " subjectivisme " peut, au moins en linguistique, y avoir aussi sa part? Suggestion qui confortait ma conviction antérieure quant aux savoirs divergents des grammairiens et des linguistes.

Celle de Benveniste se trouve en introduction d'un article republié dans ses Problèmes de linguistique générale [1962] (1966: 119, nous soulignons) :

Quand on étudie dans un esprit scientifique un objet tel que le langage, il apparaît bien vite que toutes les questions se posent [...] relativement à ce que l'on doit admettre comme fait [ital. originales], c'est-à-dire aux critères qui le définissent. Le grand changement survenu en linguistique tient précisément en ceci : on a reconnu que le langage devait être décrit comme une structure formelle, mais que cette 
description exigeait au préalable l'établissement de procédures et de critères adéquats, et qu'en somme la réalité de l'objet n'était pas séparable de la méthode propre à le définir.

17 Ce qui est aller, au moins en linguistique, bien au-delà du subjectivisme d'un simple « point de vue ». C'est affirmer qu'il n'y a pas de «faits » sans critères explicitant au préalable la manière dont on les établit, que leur «structure formelle » n'est pas dissociable de la «méthode » permettant de l'élaborer, et que, partant, elle ne peut être dégagée du réel observé par simple induction. En d'autres termes, qu'on ne peut pas observer le réel linguistique sans quelques a priori conceptuels ou théoriques.

Mes exercices de conceptualisation ont donc été conçus dans un esprit sinon constructiviste du moins "constructif», tributaire autant de mes lectures savantes d'alors que de mon expérience d'enseignant et d'apprenant ${ }^{12}$.

\section{Des exercices qui n'ont pas été toujours bien compris}

Très vite après avoir proposé ces exercices de conceptualisation, il me fallut constater que si certains enseignants de français L2 attestaient, dans leur manière de les mettre en œuvre en classe, en avoir bien saisi l'esprit constructif, plus nombreux étaient ceux qui les réduisaient à de traditionnels exercices de grammaire inductive explicitée.

Sans doute étais-je en partie responsable de cette mécompréhension, mais des discussions avec des enseignants et des spécialistes rétifs à cet esprit constructif me persuadèrent qu'il heurtait, en eux, quelque chose de plus profond, une (pré)conception, pas toujours lucidement assumée, de ce qu'est ou doit être une règle grammaticale. Il allait, pour eux, à l'encontre de l'idée qu'ils se faisaient de la «scientificité », durant ces années où «la linguistique appliquée » dominait la réflexion sur l'enseignement des langues (L1 ou L2). Une scientificité qui disqualifiait les règles grammaticales, jugées normatives, au profit de "structures ${ }^{13}$ ", présupposées immanentes à chaque langue. Bref, c'était remettre en cause le progrès des connaissances en linguistique ${ }^{14}$.

21 Ce qui nous a conduit à élaborer l'hypothèse (Besse, 1989) qu'il existait, dans l'esprit des enseignants de français L2, au moins trois (pré)conceptions de la règle grammaticale : une qui en fait un précepte à suivre, assimilant la règle du grammairien à la « loi » du juriste, que je disais partant juridique ; une qui l'assimile à ces « lois de la nature » dont Ferdinand Brunot (1919: 135) disait qu'elles «ne sont que des faits observés et généralisés », qualifi ée de descriptiviste; et une posant que la règle, tant du grammairien que du linguiste, ne résulte pas d'une induction généralisante, mais d'une démarche hypothético-déductive, que résume ainsi l'historien des sciences Kuhn (1970 [1962] : 243) : «il n'existe aucune possibilité d'expliciter le sens d'une expression comme "ce qui se trouve réellement dans la nature" en dehors d'une théorie particulière ", qualifiée de constructiviste.

Trois conceptions dont nous avons voulu vérifier, à partir d'un questionnaire portant, entre autres, sur sept «définitions » de ce que peut être une règle grammaticale - soit deux par (pré)conception et une marginale par rapport à celles-ci ${ }^{15}$-, si elles correspondaient aux représentations courantes que s'en faisaient les enseignants de franç ais L2, natifs ou non, que nous avions en formation initiale ou continue. Leurs réponses, bien que statistiquement non représentatives, attestaient globalement que la (pré)conception descriptiviste l'emportait, mais de peu dans les années 1980, sur la constructiviste, la juridique ne ralliant pas plus d'un tiers des enseignants questionnés. 

initiale de notre questionnaire ( $«$ Citez ou formulez une règle de grammaire (relative ou non à la langue française) », les réponses étaient quasi exclusivement d'ordre juridique ${ }^{16}$. Certains groupes privilégiaient nettement cette (pré)conception juridique (par exemple, tel groupe d'enseignants tunisiens de français L2), alors que d'autres s'en tenaient massivement à la (pré)conception descriptiviste (par exemple, tel groupe d'enseignants portugais de français L2). Enfin, trois quarts des réponses relevaient de représentations « mixtes » : les descriptivo-constructivistes l'emportaient sur les juridico-descriptivistes, et surtout sur les juridico-constructivistes, fortement minoritaires. Comme si le juridiste s'accordait mieux avec le descriptiviste qu'avec le constructiviste. Enfin, ces résultats étaient biaisés par l'ambiguïté de certaines «définitions ${ }^{17}$ », et par le fait que ce questionnaire était celui d'un formateur que beaucoup de questionnés savaient déjà être constructiviste $^{18}$. Si critiquables qu'ils soient, ces résultats attestent que les représentations que se font les enseignants de français $\mathrm{L} 2$ d'une règle de grammaire sont loin de coïncider, même balisées par nos définitions ${ }^{19}$, avec ce qu'en disent les dictionnaires de linguistique.

D'un point de vue constructiviste, il n'y a là rien de surprenant. Les représentations des enseignants dépendent beaucoup de la formation scolaire qui a été, antérieurement et localement, la leur, formation dont ces spécialistes ne se préoccupent guère, sauf pour s'en démarquer au nom d'une scientificité qui les distingue de celle que véhicule le système scolaire. Il s'ensuit que ces spécialistes ne tiennent guère compte des institutions et des cultures au sein desquelles ils produisent leurs savoirs savants; or ces contextes d'ordre institutionnel et culturel nous paraissent avoir joué, dans la longue durée et pour ce qui nous intéresse ici, un rôle capital dans l'émergence et les caractéristiques des trois (pré)conceptions que nous avions, sans nous y référer, distinguées ${ }^{20}$.

\section{Peut-on tout enseigner/apprendre de la « grammaire » du français L2 par des exercices de conceptualisation?}

Évidemment non, pour des raisons qui tiennent autant à ceux (celles) qui enseignent/ apprennent le français L2 qu'au grammatical de cette langue.

Certains enseignants ne sont pas spontanément disposés à pratiquer ce type d'exercices dans leurs classes, entre autres parce qu'ayant des difficultés à suivre leurs élèves sur des chemins métalinguistiques autres que les leurs, ils ont tendance à les réduire à des exercices inductifs explicités, et en tirent la conclusion que les exercices de conceptualisation ne marchent pas. Nombre d'apprenants ne se posent guère de questions d'ordre grammatical, soit parce qu'ils relèvent de traditions éducatives qui ne traitent pas, ou très marginalement, de ces questions ${ }^{21}$, soit parce que leur usage habituel des langues (L1 ou L2), y compris écrit, s'en passe communément, soit encore parce qu'ils sont réticents, au moins pour ce qui est des langues, à toute spéculation raisonnée.

Certaines règles grammaticales ne peuvent guère s'enseigner par des exercices de conceptualisation. Deux exemples. Comment enseigner la règle d'accord des participes passés avec avoir, la première qui vient à l'esprit des enseignants de français L2, sans adopter un point de vue normatif ou juridique? N'étant pas spontanément pratiquée à l'oral par la majorité des francophones, n'étant souvent respectée à l'écrit qu'a posteriori, 
pour s'auto-corriger, elle est apprise par les francophones à la manière d'une loi juridique, son énonciation magistrale étant suivie d'une jurisprudence labyrinthique. Peut-elle être enseignée autrement en français L2? Second exemple: la plupart des francophones disent spontanément je te le donne, tu me(te) le donnes, il me (te, se) le donne, mais ils disent, tout aussi spontanément, je (tu, il) le lui donne et non *je (tu, il) lui le donne. Ce qui est pourtant conforme à l'analogie des premiers exemples, et ce que disent parfois ceux (celles) qui apprennent le français en tant que L2. Peut-on enseigner cette (ir)régularité autrement que par une règle de type descriptif? Soit en se bornant à constater qu'on dit te le, me le, se le mais le lui, soit en formulant ce constat comme une simple inversion (le est après te, me, se, mais le est avant lui), soit en le formulant en termes plus savants (par ex., en termes de pronoms personnels compléments directs et indirects).

On peut certes tenter de lancer les apprenants de français L2 dans un exercice de conceptualisation sur ce point, mais on ne parviendra à un résultat quelque peu pertinent que si ces apprenants sont à même, au moins intuitivement, de distinguer le "pronom " de le «article", ainsi que le "pronom direct» de me, te, se en tant que "pronoms indirects ». En d'autres termes, si les exercices de conceptualisation permettent à certains élèves de proposer des formulations métalinguistiques qui soient plus compréhensibles à leurs pairs que celles des grammairiens ou des linguistes ${ }^{22}$, ils ne permettent pas, très généralement, d'inventer des règles qui ne l'ont pas déjà été, d'une manière ou d'une autre, par ces doctes. Ces exercices se bornent à leur permettre de tester en classe l'utilité ou non de leur propre "passé grammatical » dans l'apprentissage d'une nouvelle L2, à savoir si ce qu'ils ont déjà appris du savoir de ces doctes à propos de leur L1 ou d'une première L2, est pour eux, au niveau qui est le leur, une aide ou un obstacle dans leur appropriation progressive des régularités de cette L2. Ils ne sont donc pas susceptibles d'intéresser les apprenants qui sont sans passé grammatical d'origine savante, ou qui en ont presque tout oublié.

L'idée de ce type d'exercice n'était nouvelle au début des années 1970 que par rapport aux exercices structuraux et à ce qu'on appelait " phase d'exploitation » dans la méthodologie SGAV, telle du moins qu'elle était alors mise en œuvre. J'ai eu, dès années plus tard, le plaisir de découvrir qu'elle ne l'était guère plus dans l'histoire de la didactique des L2. Radonvilliers, dans la seconde moitié du XVIII ${ }^{e}$ siècle, propose une démarche voisine (1768: 229-230) :

D'ailleurs les élèves, à mesure qu'ils feront des progrès, découvriront eux-mêmes des principes. Car telle est la nature de l'esprit humain : dès qu'il a acquis plusieurs idées, il les combine, il les compare, il en observe les rapports; [...] Ces remarques ne se développeront pas dans leur esprit, de façon qu'ils puissent les déduire bien clairement ; mais elles y seront cependant, \& leur serviront de guide dans la lecture. Elles en composeront une grammaire personnelle, qui aura pour eux une véritable utilité, parce qu'elle sera le fruit de leurs réflexions \& non pas de celles d'autrui.

Radonvilliers n'était certes pas constructiviste, au sens qu'on prête actuellement à ce terme, en ce que pour lui les catégories grammaticales du nom, du verbe, de l'adjectif, etc. étaient des catégories de la pensée (« de l'esprit humain»), et non des outils conceptuels «construits" par les grammairiens afin d'analyser et raisonner les langues; le passé grammatical des apprenants partant n'avait donc pas à être pris en compte. En outre, et il raisonnait dans un cadre méthodologique tout autre que le SGAV, puisque les élèves se familiarisaient à la L2 par la lecture de textes doublement traduits en L1 (« version des mots » et « version de la pensée »). Mais son idée d'une « grammaire personnelle » qui a 
pour les apprenants « une véritable utilité », étant le « fruit de leurs réflexions et non pas de celles d'autrui ", est la même que celle qui sous-tend les exercices de conceptualisation.

\section{BIBLIOGRAPHIE}

Benveniste, É. (1966). Problèmes de linguistique générale. Paris : Gallimard.

Besse, H. (1970a). « Problèmes de sens dans l'enseignement d'une langue étrangère ». Langue franç aise, $\mathrm{n}^{\circ}$ 8, décembre, pp. 61-77.

Besse, H. (1970b). « Grammaire et exercice structuraux ». Voix et images du CREDIF, n 6, avril-juin, pp. 2-5.

Besse, H. (1972) [1970]. « Grammaire structurale et exercices structuraux ». Feuille d'information des professeurs de l'académie de Toulouse enseignant la langue anglaise, n 2 février, pp. 8-19. Toulouse : Annales du Centre Régional de Documentation Pédagogique de Toulouse.

Besse, H. (1974). « Les exercices de conceptualisation ou la réflexion grammaticale au niveau 2 ». Voix et Images du CREDIF, $\mathrm{n}^{\circ}$ 2, Nouvelle série, pp. 38-44.

Besse, H. (1986). «Quelques précisions sur une problématique grammaticale contestée (réponse à M. Candelier) ». Les Langues Modernes, $n^{\circ}$ 2, pp. 75-77.

Besse, H. (1989) «L'hypothèse, la règle et la loi ». Le français dans le monde. Recherches et Applications, numéro spécial, ... et la grammaire de février-mars, pp. 103-112.

Besse, H. (2005). « De la nécessité présente d'une métalinguistique contrastive ». In Mochet, M.-A. \& al. coord. Plurilinguisme et apprentissage. Mélanges Daniel Coste. Lyon : ENS Editions. pp. 71-87.

Besse, H., Porquier, R. (1984). Grammaires et didactique des langues. Paris : Hatier-Crédif.

Brunot, F. (1919) [1909]. L'enseignement de la langue française. Cours de méthodologie. Paris :

Armand Colin.

Comenius J.-A. (1992) [1657]. La Grande Didactique ou l'art universel de tout enseigner à tous. Traduction de M.-F. Bosquet-Frigout, D. Saget, B. Jolibert. Paris : Editions Klincksieck.

Kuhn, Th. S. (1972) [1962]. La structure des révolutions scientifiques. Traduction de Laure Meyer. Paris : Flammarion.

Radonvilliers, C. F. de (1768). De la maniére d'apprendre les langues. Paris : chez Saillant.

Saussure, F. de (1973) [1915]. Cours de linguistique générale. Édition critique préparée par Tullio de Mauro. Paris : Payot.

\section{NOTES}

1. Énoncé de Georges Balandier, ethno-sociologue de l'Afrique, cité par Jean Copans (Le Monde du $07 / 10 / 2016: 14)$. 
2. Structuro-Globale Audio-Visuelle, dont les promoteurs furent Georges Gougenheim, Paul Rivenc et Petar Guberina.

3. Centre de Recherche et d'Étude pour la Diffusion du Français, de l'École Normale Supérieure de Saint-Cloud.

4. On a longtemps enseigné les règles du latin en latin, pratique remise en cause dès le XVII ${ }^{\mathrm{e}}$ siècle, entre autres par Comenius pour qui c'était là « expliquer l'hébreu par l'hébreu, l'arabe par l'arabe » [1657] (Comenius, 1992 : chap. XVII, § 27).

5. Du latin implicare, littéralement « envelopper dans ", "plier dans » : le participe passé engage, même s'il est sous-entendu, un agent qui, connaissant déjà la règle, est à même de la "plier dans » des exemples ad hoc.

6. Pattern est un modèle ou un patron tels que les couturières entendent ces mots; drill est un exercice ou une manœuvre tels que les militaires l'entendent.

7. On trouvait dans les manuels des «tables de structure " privées de toute explicitation, et la consigne de ces exercices pour laboratoire s'en tenait à un « modèle » de la " substitution » ou de la «transformation » que les élèves devaient répéter.

8. Il s'agit d'un polycopié des « divers exposés faits au cours du stage consacré aux utilisateurs de Laboratoire de Langues, au C. R. D. P. de Toulouse les 5 et 6 février 1970 ».

9. Répéter mécaniquement une série de "substitutions " (Je ferai danser les filles/chanter les garçons/écouter des disques...) peut conduire des élèves à produire des phrases telles *je ferai dire les garçons/écouter les filles..., conformes à la « structure » inculquée mais inacceptables en français.

10. Il vaut mieux ne pas lancer un exercice de conceptualisation sur un point de grammaire dont on sait qu'il n'a pas été traité par les grammairiens ou linguistes tant de la L1 que de la L2, et certaines classes, relevant de tradition culturelle peu grammaticalisées, ne se posent guère de questions d'ordre grammatical.

11. J'avais suivi les cours de Georges Gougenheim, Robert-Léon Wagner, Jacqueline Pinchon et Jean-Claude Chevalier. Au premier qui me demandait, lors de l'oral de mon D.E.S. (Diplôme d'Études Supérieures), ce que je voulais faire plus tard, j'avais répondu que la grammaire m'intéressait parce que « les grammairiens étaient rarement d'accord entre eux », ce qu'il avait bien voulu approuver.

12. En Écosse, j'avais constaté que les étudiants inscrits dans un département de français (L2) ignoraient à peu près tout des catégories grammaticales servant traditionnellement à analyser cette langue. En commençant à apprendre le chinois «mandarin » par la méthode Silent Way, et donc sans aucune explication métalinguistique du maître, je m'étais entêté durant des heures à rechercher en vain des articles ou déterminants dont cette langue se passe.

13. Rappelons que Saussure n'use de ce terme qu'au singulier, au sens de système.

14. On trouve un écho de ces discussions dans Besse (1986).

15. Pour la (pré)conception juridique : « c'est un précepte pour bien parler ou bien écrire » et «c'est une formule qui prescrit comment on doit orthographier la langue»; pour la descriptiviste: «ce sont des faits observés et généralisés» et "c'est une description des régularités internes de la langue "; pour la constructiviste : «c'est une hypothèse proposée par les grammairiens au sujet d'un mécanisme de la langue » et «c'est une représentation explicitée de la manière dont peut fonctionner une langue ». À quoi, avait été ajouté « c'est ce qui gouverne nos comportements langagiers ", inspiré de la notion de rule-governed behavior relevant de la théorie des actes de langage.

16. Massivement, la règle d'accord des participes passés avec avoir et celle qui dit que « quand deux verbes se suivent le second est à l'infinitif ».

17. Par ex., la « définition » selon laquelle «c'est une représentation explicitée de la manière dont peut fonctionner une langue » est interprétable, en raison de une et de peut, soit comme constructiviste soit comme descriptiviste. 
18. J'avais publié en 1984, en collaboration avec Rémy Porquier, Grammaires et didactique des langues, dont la première partie était clairement, mais sans que le mot y soit présent, constructiviste.

19. Une question ouverte («Remarques : ) était restée quasi sans réponse.

20. Pour plus de détails, voir Besse (1989). Le titre de l'article est des coordonnateurs du numéro, lesquels n'y ont pas maintenu les références bibliographiques.

21. C'est le cas de la tradition britannique, au moins à certaines époques, et des traditions chinoise et japonaise, dont la plupart des élèves ne s'initient à des questions de grammaire qu'en apprenant l'anglais en tant que L2.

22. Par exemple, tel apprenant qui justifie qu'on ne puisse dire *il a été en train de manger ainsi : «o $\mathrm{n}$ ne peut dire en même temps c'est fini et pas fini », ou tel autre, interprétant la différence entre l'imparfait et le passé composé, en disant : "l'imparfait, c'est comme le cinéma ; le passé composé, comme la photographie ».

\section{RÉSUMÉS}

Il s'agit d'un point de vue rétrospectif sur les exercices de conceptualisation que l'auteur a proposés, dans le cadre de la méthodologie Structuro-Globale - Audio-Visuelle (SGAV), au tout début des années 1970. Y est d'abord rappelé le contexte didactique contre lequel ils ont été conçus, celui des exercices structuraux, qui relevaient de présupposés anti-cognitifs. Ceux dits de conceptualisation spéculent, à l'opposé, sur la capacité que certains apprenants ont de réfléchir sur le sens et l'acceptabilité grammaticale des phrases qu'ils sont à même, à telle étape de leur apprentissage, de produire en L2. Ils reposent sur des présupposés d'ordre constructiviste que n'admettent pas nombre d'enseignants, ce qui les conduit à en faire de simples exercices de grammaire inductive. Il s'ensuit que, pour les mettre en œuvre dans le hic et nunc de sa classe, l'enseignant se doit d'être à même de relativiser ses savoirs grammaticaux, d'admettre que ses élèves puissent raisonner autrement que lui quant à la grammaire de cette L2, et que ceux-ci disposent d'un minimum de concepts métalinguistiques, appris antérieurement à propos de leur L1 ou d'une première L2, leur permettant de construire leurs propres réflexions grammaticales sur leurs erreurs et réussites quant à leur maîtrise progressive d'une L2 qui leur est encore largement étrangère.

The author revisits the conceptualization exercises which he devised in the early 1970s in the framework of the Audio-Visual-Global-Structural (SGAV) methodology. He first recalls the didactic context in reaction to which he introduced them, namely the pattern-drills then based on anti-cognitive premises. Conversely, those exercises described as conceptualization-aimed rely on the ability of some of the learners to reflect on the meaning and grammatical acceptability of the sentences which they are able to produce at a particular stage of their learning process. The exercises in question are built on presuppositions of a constructivist order, which are not accepted by a number of teachers and may consequently end up being turned into mere exercises of inductive grammar. It follows that, in order to use them in the hic et nunc of their classrooms, the teachers must be able to relativise their grammatical knowledge, and to accept that their students may not reason like them, when confronted to the grammar of the L2, at stake as they may draw on a stock of basic grammatical notions previously acquired while learning their own L1 or an earlier L2 and are therefore able to construct their personal 
grammatical representations of their own successes and failures while coping with a still largely foreign language.

\section{INDEX}

Mots-clés : exercice de conceptualisation, exercice structural, constructivisme, grammaire inductive, grammaire implicite

Keywords : conceptualization-aimed exercises, pattern drills, constructivism, inductive grammar, implicit grammar

\section{AUTEUR}

\section{HENRI BESSE}

ENS de Lyon, France

Après avoir enseigné le français aux universités de Saint-Andrews (Ecosse) et du Caire (Egypte), Henri Besse s'est spécialisé, au Centre de Recherche et d'étude pour la Diffusion du Français (CREDIF) de l'école Normale Supérieure de Saint-Cloud (actuellement ENS de Lyon), en linguistique et didactique des langues étrangères ou secondes (L2). Il est l'auteur, en collaboration avec Rémy Porquier, de Grammaires et didactique des langues (Paris : Didier 1984), ainsi que de nombreux articles portant sur l'enseignement / apprentissage de la « grammaire » des L2. 